\title{
Findings from post-intervention analysis of pre-eclampsia/ eclampsia in Kogi State
}

Salisu Mohammed Ishaku

Population Council

Follow this and additional works at: https://knowledgecommons.popcouncil.org/departments_sbsr-rh

Part of the Demography, Population, and Ecology Commons, Family, Life Course, and Society Commons, International Public Health Commons, Maternal and Child Health Commons, and the Women's Health Commons How does access to this work benefit you? Let us know!

\section{Recommended Citation}

Ishaku, Salisu Mohammed. 2018. "Findings from post-intervention analysis of pre-eclampsia/eclampsia in Kogi State," Ending Eclampsia Research Brief. Abuja: Population Council. 
Findings from postintervention analysis of pre-eclampsia/ eclampsia in Kogi State

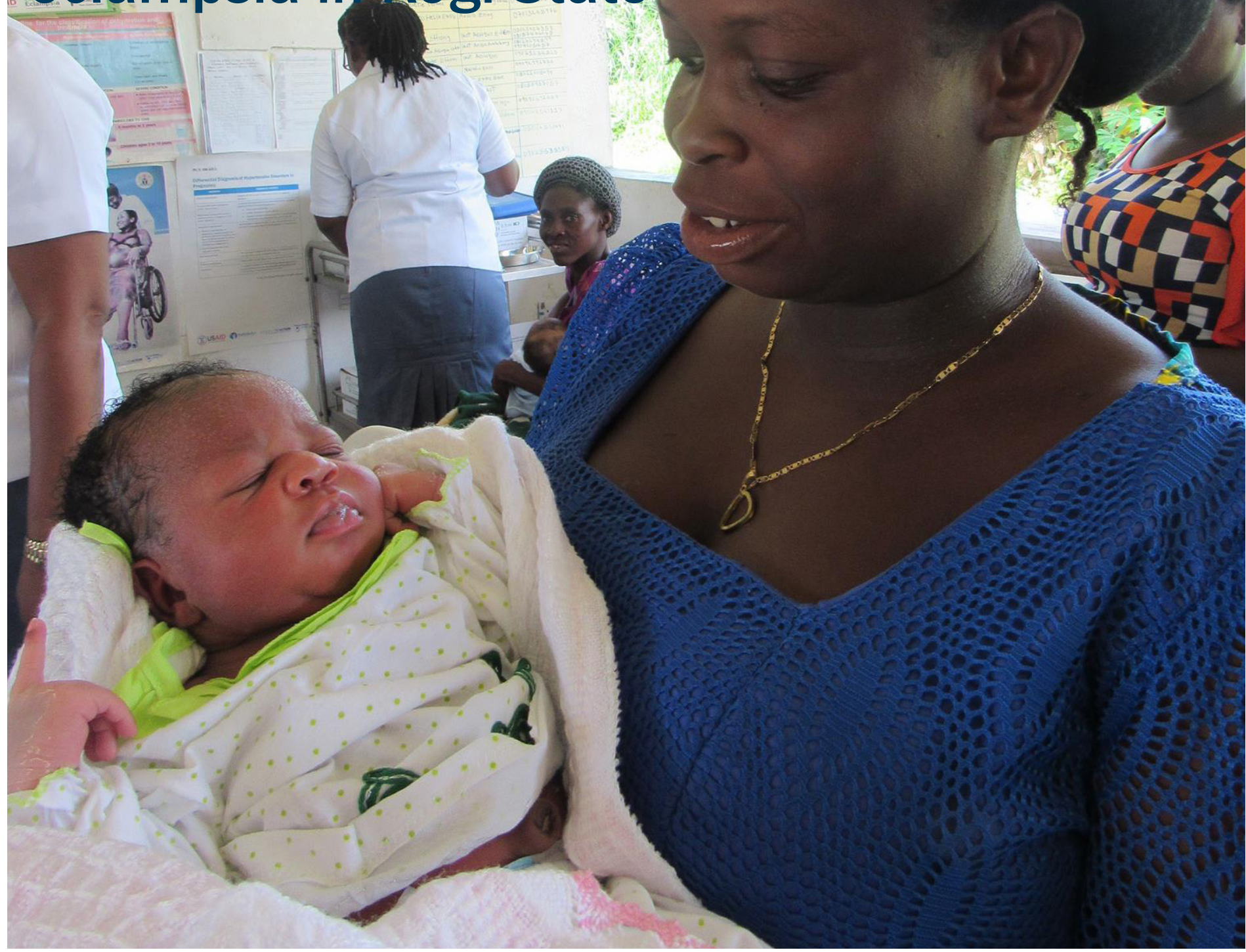

\section{USAID}

Made possible by the generous support of the American people through the United States Agency

for International Development (USAID).
The Population Council conducts research and delivers solutions that improve lives around the world. Big ideas supported by evidence: It's our model for global change. popcouncil.org

(c) 2018 The Population Council, Inc

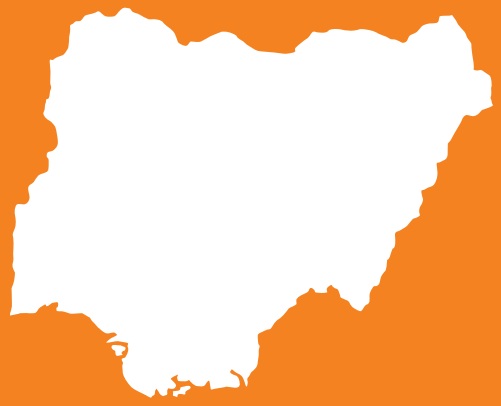

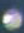

P poovuATION 


\section{BACKGROUND}

Despite global efforts to reduce preventable maternal and neonatal mortality, Nigeria's maternal mortality ratio is estimated at 576 deaths per 100,000 live births and neonatal death is estimated at 37 per 1,000 live births .

Maternal and newborn deaths due to pre-eclampsia and eclampsia (PE/E) are preventable, yet in Nigeria this is the most significant direct cause of maternal mortality.

To appreciate the enormity of this problem at country and state levels, Population Council conducted a landscape analysis on PE/E in seven states in Nigeria were to understand Nigeria's PE/E context, including assessment of evidence gaps over the last 15 years, and to determine priority areas for research and interventions around PE/E. This brief presents study findings from Kogi State on post-intervention landscape changes in:

- Programmatic and policy support for PE/E prevention and treatment;

- PHC facilities' capacity to manage PE/E;

- Provider knowledge and competence in preventing, detecting, and managing PE/E; and

- Community awareness, beliefs, and experiences around PE/E.

\section{METHODS}

This PE/E study is a pre/post, cross-sectional design with both qualitative and quantitative data collected at baseline (2015) and endline (2018). Data collection activities include in-depth interviews (IDIs) with policy makers, survivors of $\mathrm{PE} / \mathrm{E}$, and laboratory workers; focus group discussions (FDGs) with men and women from the community; and surveys and observations of client-provider interactions to assess provider capacity in antenatal care (ANC) and facility readiness and capacity to identify, treat, and manage PE/E at the primary and secondary levels.

Table 1 describes data collection activities at baseline and endline in Kogi.

\begin{tabular}{|l|c|c|}
\hline \multirow{2}{*}{ Table 1: Data collection activities in Kogi } \\
\cline { 2 - 3 } & \multicolumn{2}{|c|}{$\mathrm{n}$} \\
\hline IDIs with policy makers & 10 & Endline \\
\hline IDIs with PE/E survivors & 5 & 6 \\
\hline IDIs with laboratory professionals & - & 6 \\
\hline FGDs with married men \& women & 4 & 4 \\
\hline Surveys with providers & 74 & 73 \\
\hline Assessments of health facilities & 8 & 60 \\
\hline Observations of ANC visits & 15 & 266 \\
\hline
\end{tabular}

\section{PE/E IN BRIEF}

- $P E$ is a condition in pregnant women marked by an increase in blood pressure and protein in urine after 20 weeks gestation; eclampsia is a life-threatening condition characterized by convulsions in women with PE.

- Providing high quality ANC improves the prevention and early detection of PE and can prevent its progression to eclampsia.

- PE/E can be managed by administering anti-hypertensive drugs and $\mathrm{MgSO}_{4}$.

\section{CORE INTERVENTION}

- Intervention research occurred in Cross River, Ebonyi, and Kogi states, in 2016 and 2017, and follow-up data was collected from May-June 2018.

- 557 primary health care (PHC) providers received one month of training with continuous mentorship to identify hypertension and $P E / E$, treat with $\mathrm{MgSO}_{4}$, and refer to secondary facilities.

- The primary objective was to facilitate task-shifting to $\mathrm{PHC}$ providers of $\mathrm{MgSO}_{4}$ loading dose administration and secondary facility referral for improved PE/E detection, treatment, and management.
The Ending Eclampsia project seeks to expand access to proven, underutilized interventions and commodities for the prevention, early detection, and treatment of pre-eclampsia and eclampsia and strengthen global partnerships. 


\section{POLICY INFLUENCE}

At endline, researchers interviewed 28 local government area (LGA) and state policy makers revealing mixed awareness of the national protocol on managing $\mathrm{PE} / \mathrm{E}$ through task shifting to PHC providers. Though more local policy makers knew of the national protocol than at baseline, at least one LGA health manager did not know whether task shifting had been implemented in their area. Those who are aware of the protocol support task shifting to lower level cadres for PE/E management.

\section{"In general, [task-shifting and referral] is very good because it will help the pregnant women, it will help our patients."}

\section{- LGA health manager, 2017}

Policy makers are concerned about pregnant women registering late for ANC as a principle challenge in detecting and managing PE/E. Policy makers cite poverty and lack of information about ANC as barriers to women's care-seeking behaviors.

\begin{abstract}
"Beginning with the antenatal services, some of them do not have the financial ability to access the services and some other persons, due to their literacy level, do not see the need to attend antenatal clinics. Some of the husbands do not allow their wives to attend antenatal clinics, and many of them do not keep to their appointments. They miss appointments due to one reason or the other."
\end{abstract}

- LGA policy maker, 2018

\section{FACILITY CAPACITY \& PREPAREDNESS}

At baseline, researchers visited eight health facilities in Kogi and found that only one (13\%) had guidelines available to manage $\mathrm{PE}$, one had all vital ANC equipment, and four consistently used $\mathrm{MgSO}_{4}$ to treat PE/E. At endline, researchers visited 48 primary and 12 secondary healthcare facilities and observed that 56 (93\%) have available guidelines to manage PE $(p<0.001)$.

At baseline, only one out of eight facilities (13\%) had all the necessary ANC equipment to detect PE/E; at endline, five out of 14 facilities (36\%) had all equipment. At baseline, $75 \%$ of facilities reported capacity to procure $\mathrm{MgSO}_{4}$ and, of those, $50 \%$ asked clients to purchase the drug themselves. At endline, $59 \%$ of facilities purchased $\mathrm{MgSO}_{4}$ from local pharmacies, while only $13 \%$ asked clients to procure it themselves.

Though not statistically significant, the decrease in percent of clients who were asked to procure $\mathrm{MgSO}_{4}$ themselves indicates that facilities are now better able to procure the drug than at baseline.

Eighty-one percent of facilities at endline had $\mathrm{MgSO}_{4}$ available on-site, compared to only $25 \%$ at baseline. Four (50\%) facility managers said they always used $\mathrm{MgSO}_{4}$ at baseline, and at endline 22 (42\%) managers reported always using $\mathrm{MgSO}_{4}$. At baseline, two (25\%) facility managers said they sometimes use $\mathrm{MgSO}_{4}$ to manage $\mathrm{PE} / \mathrm{E}$, while at endline 12 (23\%) report sometimes using it. Neither of these changes was statistically significant and percent decreases are likely due to sample size differences.

Figure 1: Facilities with key equipment to detect and manage $\mathrm{PE} / \mathrm{E}$ and $\mathrm{MgSO}_{4}$ toxicity (\%)

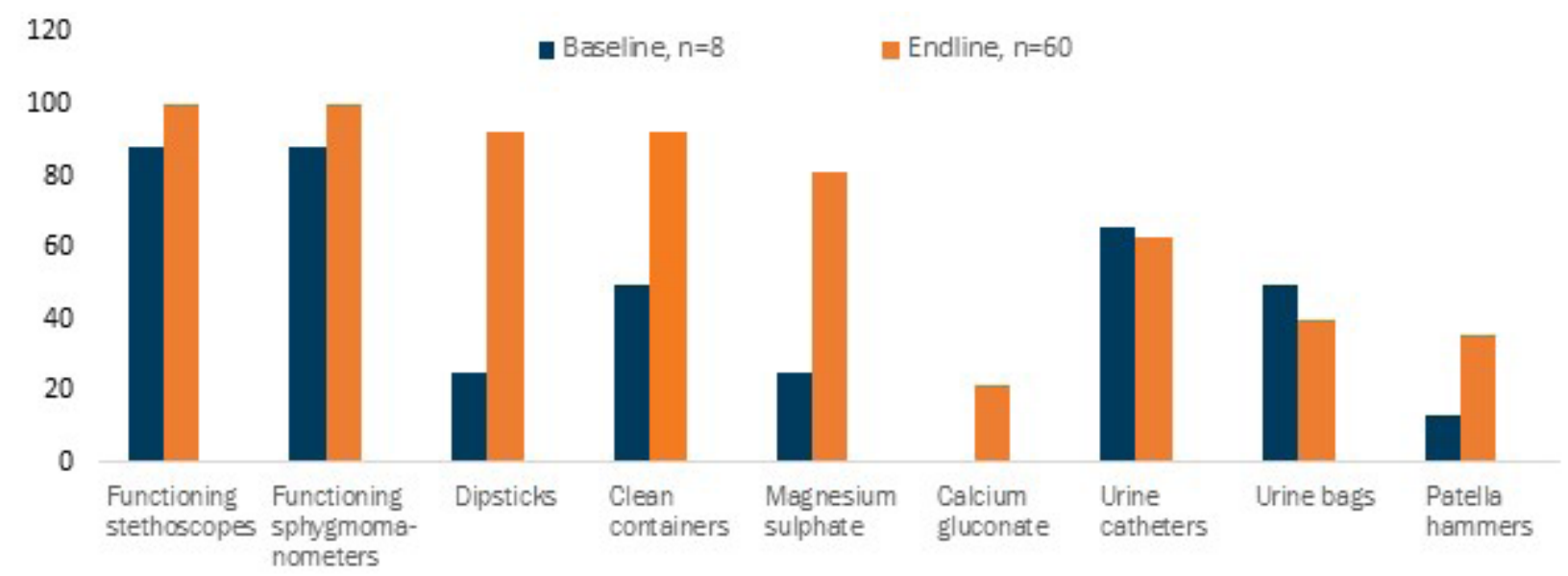


Researchers identified the number of facilities that stocked key equipment to detect and manage PE/E and treat $\mathrm{MgSO}_{4}$ toxicity. Improvements were observed at endline for stocking all key equipment except urine bags (Figure 1).

A critical component of managing and treating severe $\mathrm{PE} / \mathrm{E}$ is the capacity to conduct emergency obstetric and neonatal care (EmONC) by providing key signal functions and essential drugs to mothers throughout the pregnancy continuum (pregnancy, labor and delivery and postnatal).

Of all facilities visited in Kogi State at endline, 14 (23\%) stock all essential equipment, and $36(60 \%)$ stock at least three-quarters of the essential supplies. Table 2 compares facilities' capacity to provide EmONC signal functions at baseline and endline.

\begin{tabular}{|c|c|c|c|c|}
\hline & \multicolumn{2}{|c|}{ Baseline, $n=8$} & \multicolumn{2}{|c|}{ Endline, $n=60$} \\
\hline & Capacity & $\begin{array}{l}\text { Done } \\
\text { in past } \\
3 \text { mos }\end{array}$ & Capacity & $\begin{array}{l}\text { Done } \\
\text { in past } \\
3 \text { mos } \\
\end{array}$ \\
\hline $\begin{array}{l}\text { Perenteral } \\
\text { antibiotics }\end{array}$ & 7 & 6 & 56 & 52 \\
\hline Parenteral oxytocics & 6 & 7 & 56 & 52 \\
\hline $\begin{array}{l}\text { Parenteral } \\
\text { anticonvulsants } \\
\left(\mathrm{MgSO}_{4}\right)\end{array}$ & 7 & 6 & 43 & 26 \\
\hline $\begin{array}{l}\text { Manual placenta } \\
\text { removal }\end{array}$ & 8 & 7 & 50 & 31 \\
\hline $\begin{array}{l}\text { Retained products } \\
\text { removal }\end{array}$ & 6 & 6 & 43 & 26 \\
\hline $\begin{array}{l}\text { Assisted vaginal } \\
\text { delivery }\end{array}$ & 7 & 7 & 38 & 33 \\
\hline Blood transfusion & 7 & 7 & 22 & 19 \\
\hline Caesarean section & 6 & 6 & 12 & 10 \\
\hline $\begin{array}{l}\text { Neonatal } \\
\text { rescuscitation }\end{array}$ & 7 & 6 & 52 & 42 \\
\hline
\end{tabular}

\section{PROVIDER KNOWLEDGE \& SKILLS}

At endline, 73 providers from primary and secondary facilities in Kogi State responded to questions about preventing, detecting, and treating PE/E. Providers can correctly identify and manage hypertension in pregnancy.

Ninety percent of providers correctly diagnosed hypertension at endline compared to $86 \%$ at baseline, and significantly more providers use antihypertensive drugs to treat hypertension at endline compared to baseline ( $85 \%$ vs. $45 \%$; $p<0.001)$.
Figure 2: Providers' knowledge of drugs for PE/E prevention and management (\%)

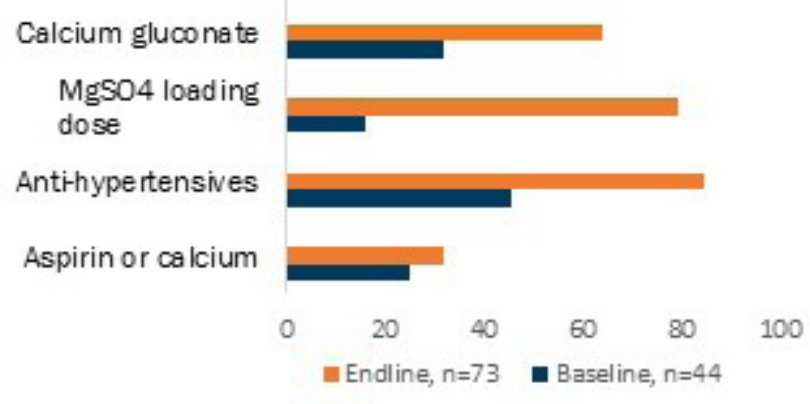

A key component of task shifting from secondary to primary facilities is PHC providers' capacity to prevent and manage $\mathrm{PE} / \mathrm{E}$ using antihypertensive drugs, $\mathrm{MgSO}_{4}$, and calcium gluconate to treat $\mathrm{MgSO}_{4}$ toxicity.

Figure 2 highlights knowledge improvement among PHC providers from baseline to endline.

This intervention is based on the Pritchard method for administering loading and maintenance doses of $\mathrm{MgSO}_{4}$ to treat severe pre-eclampsia and prevent the onset of eclampsia. At baseline, $16 \%$ of providers could correctly describe the loading dose of $\mathrm{MgSO}_{4}$ and none knew the appropriate maintenance dose. At endline, $79 \%$ know the appropriate loading dose $(p<0.001))$ and more than half $(52 \%)$ know the correct maintenance dose $(p<0.001)$.

Similarly, the number of providers who know the correct antidote to $\mathrm{MgSO}_{4}$ toxicity doubled from baseline to endline ( $32 \%$ to $64 \%$, respectively, $p=0.001$ ). Kogi State was the only state where the number of providers able to correctly identify pre-eclampsia decreased (from 95\% to $67 \% ; p<0.001$ ), though this may be attributed to a shorter intervention period in Kogi.

\section{QUALITY OF CARE}

Quality of care was assessed through 266 observations of client-provider interactions during ANC consultations, qualitative data from four men's and women's community FGDs, and six interviews with PE/E survivors. Figure 3 compares the proportions of ANC components that clients received at baseline and endline. At baseline, virtually all clients had their blood pressure checked during the observed ANC visits. However, 76 clients (30\%) did not have their urine tested for protein.

Clients' reports confirm findings from Figure 3 that BP, proteinuria, and weight screenings often occur during ANC. In an important contrast to baseline findings where no providers were observed discussing the symptoms of 


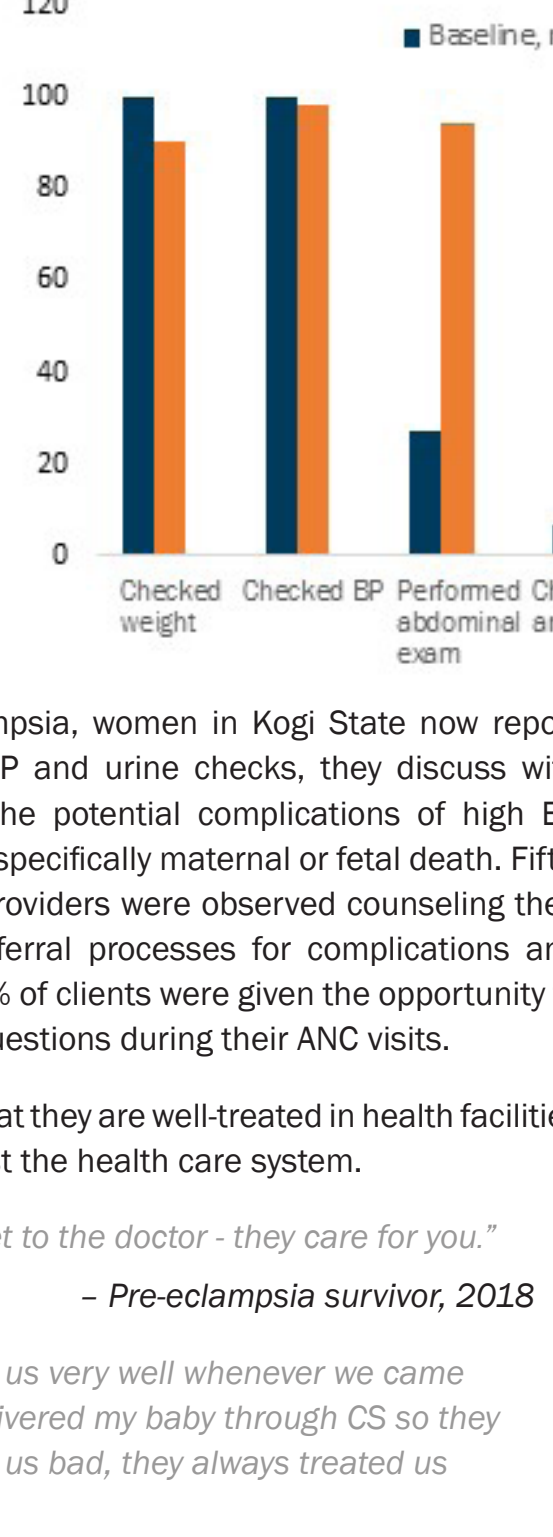

- Pre-eclampsia survivor, 2018

Laboratory staff report mixed experiences with supply stocks. While some facilities experience infrequent stock outs of dipsticks for proteinuria, likely due in part to regular oversight and procurement procedures, other facilities experience frequent stock outs and delayed replenishments.

\footnotetext{
"The materials, the laboratory consumables, the reagents we are using in the laboratory most of the problem we are having is from that area sometimes you write a request out for reagent it may take you about a month before you will be able to get the reagent pack."

- Laboratory department head, PHC, 2018
}

\section{COMMUNITY KNOWLEDGE \& PERCEPTIONS}

Community knowledge and perceptions of hypertension and related complications during pregnancy are mixed. Similar to baseline, men and women attribute the symptoms of hypertension to a woman's over-thinking and fear, and religious leaders cite spiritual attack. Interestingly, men in Kogi State perceive the cause of hypertension to be a woman's failure to attend ANC, while women (both FGD participants and PE/E survivors) overwhelmingly state that most women in Kogi State seek health services from ANC providers.

Some community members describe a woman's husband and mother-in-law as encouraging her to seek facilitybased care, while others say that husbands and mothersin-law may dismiss hypertension-related symptoms as signs of laziness or a woman's "pretending."

At endline, community members report that some women seek facility-based care, while others continue to use herbal medications. Men and women in the community cite financial concerns as the primary barrier to women's ANC attendance, though most survivors of $\mathrm{PE} / \mathrm{E}$ say that finances were not a concern in her decision to go to the hospital for care. At endline, men and women have differing perceptions of religious leaders' roles in the health system. Women report that religious leaders will simply pray for a woman's health, 
while men reported religious leaders act as barriers along the referral pathway by delaying women's seeking care at facilities.

"At times pastor tell them that they should be coming for prayer instead of advising them to visit the hospital for proper check-up. They will be deceiving them to be coming for prayer. That one will discourage them to visit the hospital."

- Male FGD participant, 2018

\section{SURVIVOR'S EXPERIENCES}

While most survivors attended ANC, the timing and reasons for seeking care varied widely. One woman began ANC at two months to verify that she was pregnant and subsequently attended at least 10 ANC check-ups, while another survivor registered for ANC during her 8th month because she was experiencing headache and other symptoms, which she believed were due to malaria.

Survivors consistently report that providers check their $\mathrm{BP}$ and urine, though BP test results are more frequently discussed than urine. A few women state their providers discussed with them the potential consequences of high BP or proteinuria during, including convulsion, and recommended a cesarean section (CS) delivery.

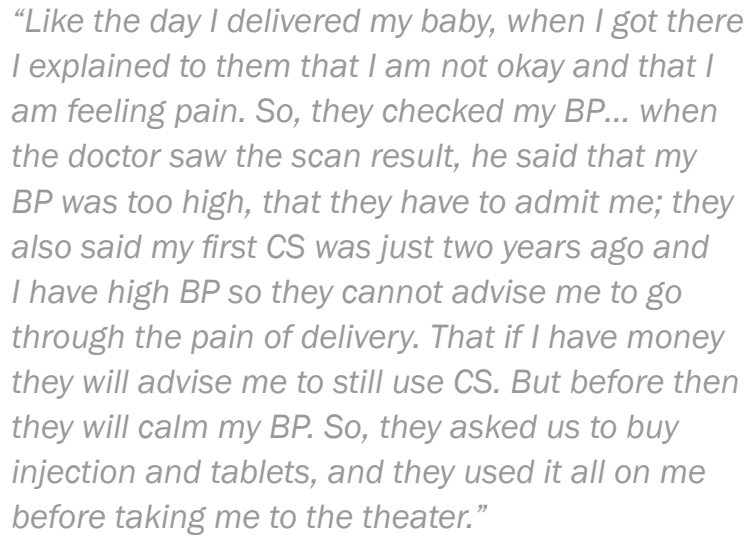

- Pre-eclampsia survivor, 2018

\section{DISCUSSION}

Policy makers recognize the importance of scaling up taskshifting of $\mathrm{MgSO}_{4}$ loading dose administration and referral to PHC providers, and see it as a means of overcoming barriers to hospital attendance. Facility capacity to detect, treat, and manage PE/E has improved. Fewer women are responsible for procuring their own $\mathrm{MgSO}_{4}$, which contributes to improving facility accessibility and affordability. More facilities in Kogi stocked all or most key equipment for detecting and managing pre-eclampsia and $\mathrm{MgSO}_{4}$ toxicity than in any other state.
Sufficient supply chain commodities have implications for provider capacity, and provider capacity in Kogi State improved significantly when identifying and managing women with hypertension. Conversely, providers at endline were less able to correctly identify PE/E. This may be linked to the fact that Kogi State remained the comparison, non-intervention state for implementation. Focused re-training on $\mathrm{PE} / \mathrm{E}$ identification at the community level, or supplemental training at the $\mathrm{PHC}$ level like those that occurred in Cross River and Ebonyi states, will further improve providers' diagnostic knowledge retention and women's uptake of care.

Gender-based and intergenerational differences in community and survivor perceptions of key components of pregnancy care may affect ANC attendance and response to symptoms. Mixed use of traditional and facility-based health services and low integration of religious leaders into the health system offer opportunities for continued health education and community collaboration.

One limitation of the data is the unequal sample sizes from ANC observations at baseline and endline. The duration of the intervention was relatively short (14 months); a longer period may have demonstrated further increase in provider knowledge and confidence in practices around $\mathrm{PE} / \mathrm{E}$ care. However, triangulating across different data sources and types renders our study findings robust.

\section{RECOMMENDATIONS}

- Sustain support for supply chain procurement and linkages to existing distribution system;

- Re-train providers at primary and secondary levels to correctly identify pre-eclampsia, incorporating antihypertensive drugs training that was previously implemented in Ebonyi State;

- Continue mentoring efforts and supportive supervision for PHC providers to detect and manage hypertensin in pregnancy, including PE/E; and

- Expand community health education about the causes, signs, symptoms, and complications of hypertension during pregnancy, with particular emphasis given to engaging pre-existing women's and other community groups.

\section{REFERENCES}

\section{DHS Nigeria, 2013.}

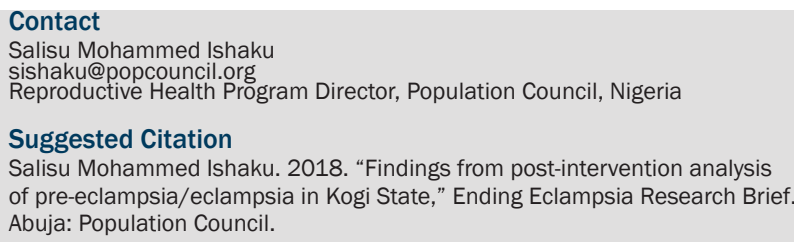

